Pacific Journal of Mathematics

FINITE-DIMENSIONAL PERTURBATION AND A
REPRESENTAION OF SCATTERING OPERATOR 


\title{
FINITE-DIMENSIONAL PERTURBATION AND A REPRESENTATION OF SCATTERING OPERATOR
}

\author{
S. T. KURODA
}

1. Introduction. In what follows we shall be concerned with the problem of perturbation of continuous spectra by an operator of finite rank. Namely, we consider two self-adjoint operators $H_{0}$ and $H_{1}$ in a Hilbert space $\mathfrak{S}$ which are related to each other as follows:

$$
\left\{\begin{array}{l}
H_{1}=H_{0}+V, \\
V x=\sum_{k=1}^{r} c_{k}\left(x, \varphi_{k}\right) \varphi_{k}, \quad x \in \mathfrak{W},
\end{array}\right.
$$

where $\left(\varphi_{i}, \varphi_{j}\right)=\delta_{i j}$ and $c_{k}$ is a nonzero real number. For this problem the existence of the so-called wave operator $W_{ \pm}$and the scattering operator ${ }^{1} S$ was proved by Kato [3] together with the unitary equivalence of the absolutely continuous (abbr. a.c.) parts of $H_{0}$ and $H_{1}$. As the first step of his proof, he considered the case of $r=1$ in detail and proved a sort of explicit formulas for $W_{ \pm}$and $S$ (or, in other words, representations of them in certain spectral representation spaces associated with $H_{0}$ and $H_{1}^{2}$ ).

One of the main purposes of the present paper is to give a similar kind of formulas for $W_{ \pm}$and $S$ in the case of an arbitrary finite value of $r$. For this purpose we use two kinds of spectral representation spaces. The first is the classical one due to Hellinger and Hahn (see [7, Chapt. VII]) and the second is one of its versions suitable for our problem.

For a self-adjoint operator $H$ in $\mathfrak{S}$ and a finite subset $\left\{u_{k} ; k=1, \cdots, n\right\}$ of $\mathfrak{S}$, we denote by $\mathfrak{L}\left(u_{1}, \cdots, u_{n} ; H\right)$ the smallest closed subspace of $\mathfrak{S}$ containing $\left\{u_{k}\right\}$ and reducing $H$. Then, it is known $[3, \S 2]$ that $\mathfrak{S}_{0} \equiv \mathfrak{R}\left(\varphi_{1}, \cdots, \varphi_{r} ; H_{0}\right)=\mathfrak{L}\left(\varphi_{1}, \cdots, \varphi_{r} ; H_{1}\right)$ and that $H_{0}=H_{1}$ in the orthogonal complement $\mathfrak{S}_{0}^{\prime}$ of $\mathfrak{K}_{0}$. As easy consequences, this yields that $W_{ \pm}$and $S$ are reduced by $\mathfrak{S}_{0}^{\prime}$ and that, on $\mathfrak{S}_{0}^{\prime}$, they are simply the orthogonal projection on the a.c. subspaces of $\mathfrak{S}_{0}^{\prime}$ with respect to $H_{0}$. Hence, in the study of a representation of $W_{ \pm}$and $S$, we can assume without essential loss of generality that

$$
\mathfrak{S}=\mathfrak{S}_{0}=\mathfrak{L}\left(\varphi_{1}, \cdots, \varphi_{r} ; H_{p}\right), \quad p=0,1 .
$$

Received November 14, 1962, and in revised form May 28, 1963. Research on this paper was partly supported by the National Science Foundation Grant GP-2.

1 For the exact definition of $W_{ \pm}$and $S$, see (2.1) below.

2 Certain similar representations were given in the scattering problems associated with partial differential operators of Schroedinger type. See, e.g. [2]. 
For the sake of brevity of the exposition, we assume this in the sequel. All of our theorems can be applied to the general case of $\mathfrak{K} \neq \mathfrak{S}_{0}$ with slight changes of words. This assumption being in effect, the multiplicies of the spectra (see [7, Chapt. VII]) of $H_{0}$ and $H_{1}$ are finite. Since $W_{ \pm}$furnishes the unitary equivalence under consideration, it should be represented by a matrix-valued function of the spectral parameter $\lambda$ which maps the spectral representation space of $H_{0}$ into that of $H_{1}$. In Theorem 2 we shall show that, in the representation of the second kind, this matrix function can be obtained as the boundary values $W_{ \pm}(\lambda)$ on reals of a matrix function $W(z)$ of a complex variable. The function $W(z)$, which is given in terms of $V$ and the resolvent of $H_{0}$, is regular in the resolvent set of $H_{0}$. The determinant of $W(z)$, usually called the Weinstein determinant, has been shown to have a close relation to the change of eigenvalues by the perturbation $V$ given in (1.1) (see, e.g., [4]). It might therefore be of interest to point out that the boundary values of the same matrix has certain connections with the perturbation of continuous spectra.

The representation of $S$ is readily obtained from that of $W_{ \pm}$and has the form of a matrix function $S(\lambda)$ operating in the representation space of $H_{0}$ (Theorem 2). Moreover, in a suitably chosen representation spaces of the first kind, this matrix function has a diagonal form for all values of $\lambda$. In Theorem 3, we shall give a rule of getting all of these eigenvalues of $S(\lambda)$ from that of the matrix $\left(W_{+}^{-1} W_{-}\right)(\lambda)$. Here, we note that a relation between the determinant of $S(\lambda)$ and the Weinstein determinant has recently been obtained by Birman and Krein [1] under a milder condition on $V$.

The method in the present paper may be characterized as a "timeindependent" method, in which one wants to minimize the use of the theory of wave operators. Nevertheless, in 5 and 6 we shall make free use of the already established facts on the wave operators for the sake of brevity. In compensation, a comment will be made to indicate how to pursue a "time-independent" method.

As a by-product, we shall give in 4 a seemingly new "timeindependent" proof of the unitary equivalence of the a.c. parts of $H_{0}$ and $H_{1}$. When $r=1$, this proof is the same as Kato's [3].

2. Notation. We denote by $C^{n}$ the unitary space of all $n$-tuples of complex numbers and by $C_{n}^{m}$ the subspace of $C^{n}$ consisting of all the tuples whose last $n-m$ components are zero. The inner product in $C^{n}$ is denoted by $(,)_{n}$, while that in $\mathfrak{S}$ simply by $($,$) . The$ letter $\lambda$ denotes always a real number or a real variable and ' attached to a function of $\lambda$ indicates the differentiation with respect to $\lambda$. By $M(m, n)$ we denote the set of all matrices of complex numbers having 
$m$ rows and $n$ columns. We write $M(n, n)=M(n)$. The identity in $M(n)$ is specified by the notation $I_{n}$. A matrix $A \in M(m, n)$ is sometimes regarded to be an operator from $C^{n}$ into $C^{m}$. Then $\mathfrak{R}(A)$ and $\mathfrak{R}(A)$ denote the range and the null space of the operator $A$. The same notations will be used for operators in Hilbert spaces. $\quad r(A)=\operatorname{dim} \Re(A)$ is the rank of $A$. The symbol * indicates Hermitian adjoint matrix or adjoint operator.

For a function $\sigma(\lambda),-\infty<\lambda<\infty$, of bounded variation, the symbol $d \sigma$ is provisionally used to denote the Lebesgue-Stieltjes measure determined by $\sigma$. The $L^{2}$-space over the measure $d \sigma$ is denoted by $L^{2}(\sigma)$.

Whenever the function $a_{i j}(\lambda), 1 \leqq i \leqq m, 1 \leqq j \leqq n$, are given, the matrix $\left(a_{i j}(\lambda)\right) \in M(m, n)$ whose $(i, j)$ element is $a_{i j}(\lambda)$ will be denoted by the corresponding capital letter as $A(\lambda)$. This convention may be used in the reverse way.

For a self-adjoint operator $H$ in $\mathscr{S}$ and a function $f(\lambda),-\infty<\lambda<\infty$, the operator $f(H)$ is defined according to the usual rule of the operational calculus.

The letter $p$ used as sub-(or super-) script always ranges over 0 and 1. When $p$ and $q$ are used in a certain formula, $q$ always denotes one of 0 and 1 which is different from $p$.

Finally, for the convenience of the later reference, we shall recall the definition of $W_{ \pm}$and $S$ :

$$
\left\{\begin{array}{l}
W_{ \pm}\left(H_{q}, H_{p}\right)=\underset{t \rightarrow \pm \infty}{\operatorname{sim}} \exp \left(i t H_{q}\right) \exp \left(-i t H_{p}\right) P_{p}, \\
S=W_{+}\left(H_{0}, H_{1}\right) W_{-}\left(H_{1}, H_{0}\right)=W_{+}\left(H_{1}, H_{0}\right)^{*} W_{-}\left(H_{1}, H_{0}\right),
\end{array}\right.
$$

where $P_{p}$ is the orthogonal projection on the a.c. subspace of $\mathfrak{g}$ with respect to $H_{p}$.

3. Preliminary on spectral representations. Since the argument of this section will be applicable to both $H_{0}$ and $H_{1}$, we omit the subscript 0 and 1 in this section. Let $\mathfrak{M}$ be the a.c. subspace of $\mathscr{L}$ with respect to $H=\int \lambda d E(\lambda)$ and $P$ the orthogonal projection on $\mathfrak{M}$. For later use, we first define

$$
\left\{\begin{array}{lll}
\beta_{i j}(\lambda)=\left(E(\lambda) \varphi_{j}, \varphi_{i}\right), & & 1 \leqq i, j \leqq r \\
\gamma_{i j}(\lambda)=\left(E(\lambda) P \varphi_{j}, P \varphi_{i}\right), & & 1 \leqq i, j \leqq r
\end{array}\right.
$$

The corresponding $M(r)$-valued functions are denoted by $B(\lambda)$ and $\Gamma(\lambda)$ according to our convention. Incidentally, we note the relation

$$
B^{\prime}(\lambda)=\Gamma^{\prime}(\lambda) \text {, a.e. , }
$$

which follows immediately from the definition. 
In what follows we shall construct two representations of $\mathfrak{M}$ by certain "function spaces" in such a way that $H P$ has a "diagonal" form. The assumption (1.2) implies that $\mathfrak{M}=\mathfrak{L}\left(P \varphi_{1}, \cdots, P \varphi_{r} ; H\right)$. Hence, according to the theory of self-adjoint operators in a Hilbert. space (see [7, Chapt. VII]), there exist a nonnegative integer $n \leqq r$ and $\psi_{k} \in \mathfrak{M}, 1 \leqq k \leqq n$, such that:

(i) $\left\|\psi_{k}\right\|=1$;

(ii) if $i \neq j,\left(E(\lambda) \psi_{i}, \psi_{j}\right)=0$ for every $\lambda$;

(iii) if $i>j$, the measure $d\left(E(\lambda) \psi_{i}, \psi_{i}\right)$ is absolutely continuous. with respect to the measure $d\left(E(\lambda) \psi_{j}, \psi_{j}\right)$; and

(iv) $\mathfrak{M}=\sum_{k=1}^{n} \oplus \mathfrak{L}\left(\psi_{k} ; H\right)$. The number $n$ is uniquely determined by the properties (i)-(iv), while the set $\left\{\psi_{k}\right\}$ is not. For brevity we write $\sigma_{k}(\lambda)=\left(E(\lambda) \psi_{k}, \psi_{k}\right)$ and denote by $\Sigma(\lambda) \in M(n)$ the diagonal matrix whose diagonal elements are $\sigma_{k}(\lambda)$.

As the representation space of the first kind, we take the Hilbert. space $\widetilde{F}=\sum_{k=1}^{n} \oplus L^{2}\left(\sigma_{k}\right)$. A generic element of $\widetilde{F}$ is written as $f=$ $\left(f_{1}, \cdots, f_{n}\right)$, where $f_{k} \in L^{2}\left(\sigma_{k}\right)$. The norm of $f$ is given by $\|f\|^{2}=$ $\sum\left\|f_{k}\right\|^{2}$. Occasionally, it is convenient to use a representative function. $f(\lambda)$ of $f \in \mathfrak{F}$. Precisely, a $C^{n}$-valued function $f(\lambda)=\left(f_{1}(\lambda), \cdots, f_{n}(\lambda)\right)$, $-\infty<\lambda<\infty$, is a representative function of $f=\left(f_{1}, \cdots, f_{n}\right) \in \mathfrak{F}$, if each $f_{k}(\lambda)$ is a representative function of $f_{k} \in L^{2}\left(\sigma_{k}\right)$.

By virtue of the properties (i) and (iv) of $\left\{\psi_{k}\right\}$ mentioned above, we now see that there exists a unitary map $F$ from $\mathfrak{M}$ onto $\mathfrak{F}$ which satisfies the following relationship:

$$
x=\sum_{k=1}^{n}(F x)_{k}(H) \psi_{k}, \quad \text { for each } x \in \mathfrak{M},
$$

where $F x=\left((F x)_{1}, \cdots,(F x)_{n}\right)$ is the image of $x$ by $F$. Furthermore, the mapping $F$ is uniquely determined by the requirement (3.3). In terms of the representative functions the inner product of $x, y \in \mathfrak{M}$ is. written as

$$
(x, y)=\int_{-\infty}^{\infty}\left(\Sigma^{\prime}(\lambda)(F x)(\lambda),(F y)(\lambda)\right)_{n} d \lambda, \quad x, y \in \mathfrak{M} .
$$

Furthermore, $H$ is diagonal in the sense that $(F H x)(\lambda)=\lambda(F x)(\lambda)$ for each $x \in \mathfrak{M}$ which is in the domain of $H$. (Precisely speaking, this. relation means that for each representative function $(F x)(\lambda)$ of $F x$, the right-hand side gives a representative function of $F H x$.)

One of the important properties of the matrix $\Sigma^{\prime}(\lambda)$ introduced above is that its rank is equal to the multiplicity ([7, Chapt. VII]) $m(\lambda)$ of $\lambda$ with respect to the a.c. part $H P$ of $H$. More precisely, we have the following lemma whose proof is obvious.

LEMma 3.1. Let $m(\lambda)$ be as above. Then, for almost all $\lambda$ we 
have the following fact. The derivative $\sigma_{k}^{\prime}(\lambda)$ exists for all $k$ and. $\sigma_{k}^{\prime}(\lambda)=0$ for all $k>m(\lambda)$ while none of the other $\sigma_{k}^{\prime}(\lambda)$ is zero. Therefore, $\Sigma^{\prime}(\lambda)$ maps $C_{n}^{m(\lambda)}$ onto itself and its orthogonal complement into $\{0\}$.

The representation introduced above has the advantage that the base $\left\{\psi_{k}\right\}$ is chosen to be "orthogonal". In handling the representation of $W_{ \pm}$, however, it is helpful to have another representation of $\mathfrak{M}$ which stems from $\varphi_{k}$ more directly than the first one. As is easily seen from the relation $\mathfrak{M}=\mathfrak{L}\left(P \varphi_{1}, \cdots P \varphi_{r} ; H\right)$, the space $\mathfrak{M}$ is the closure of all elements $x \in \mathfrak{M}$ which are expressible as

$$
x=\sum_{k=1}^{r} x_{k}(H) P \varphi_{k}, \quad x_{k} \in L^{2}\left(\gamma_{k k}\right) \text {. }
$$

Roughly speaking, the new representation of $x$ will be the $r$-tuple formed by $x_{k}$. In general, however, these $x_{k}$ are not uniquely determined. For this reason, we have to consider the equivalence classes of $r$-tuples, regarding those two tuples that give the same $x$ by (3.5) to be equivalent. The precise argument will be carried out by considering the relation between two expressions (3.3) and (3.5) for $x$.

For brevity we write $t_{k i}=\left(F P \varphi_{i}\right)_{k} \in L^{2}\left(\sigma_{k}\right)$. Then, (3.3) yields that

$$
P \varphi_{i}=\sum_{k=1}^{n} t_{k i}(H) \psi_{k}, \quad i=1, \cdots, r .
$$

An $M(n, r)$-valued function $T(\lambda),-\infty<\lambda<\infty$, is called a representative function of $\left(t_{k i}\right)$, if the $(k, i)$ element $t_{k i}(\lambda)$ of $T(\lambda)$ is a representative function of $t_{k i} \in L^{2}\left(\sigma_{k}\right)$. In the sequel $T(\lambda)$ serves as a transformation matrix between our representations of $\mathfrak{M}$. Incidentally, we note that there exists a representative function $T(\lambda)$ which satisfies

$$
t_{k i}(\lambda)=0 \quad \text { whenever } \sigma_{k}^{\prime}(\lambda)=0 .
$$

LEMma 3.2. (i) Let $T(\lambda)$ be a representative function of $\left(t_{k i}\right)$. Then, we have

$$
\begin{gathered}
\Gamma^{\prime}(\lambda)=\left(T^{*} \Sigma^{\prime} T\right)(\lambda), \quad \text { a.e. }, \\
\Re(T(\lambda)) \supset C_{n}^{m(\lambda)}, \quad \text { a.e. }
\end{gathered}
$$

If $T(\lambda)$ satisfies (3.7), then the equality holds in (3.9) instead of the inclusion.

(ii) The multiplicity $m(\lambda)$ of $\lambda$ with respect to the a.c. part of $H$ is given by

$$
m(\lambda)=r\left(\Gamma^{\prime}(\lambda)\right)=r\left(B^{\prime}(\lambda)\right), \text { a.e. }
$$

Proof. The formula (3.8) is a straightforward consequence of 
(3.6). For the proof of (3.9) we note that the set of all $x \in \mathfrak{M}$ which are expressible as (3.5) with some $x_{k} \in L^{2}\left(\gamma_{k k}\right)$ is dense in $\mathfrak{M}$. Then, (3.9) follows from Lemma 3.1 without much difficulty. The details may be omitted. The remaining part of (i) follows from (3.9) at once. Since $T^{*}$ is one-to-one on $\Re(T)$, we see by (3.8), (3.9), and Lemma 3.1 that $r\left(\Gamma^{\prime}(\lambda)\right)=r\left(\Sigma^{\prime}(\lambda)\right)=m(\lambda)$, which proves $(3.10)$.

We need one more matrix in the sequel. Let $Q(\lambda) \in M(n)$ be the orthogonal projection in $C_{n}$ onto $C_{n}^{m(\lambda)}$. Then, the relation (3.9) tells us that there exists a measurable $M(r, n)$-valued function $U(\lambda)$ satisfying the relation

$$
T(\lambda) U(\lambda)=Q(\lambda), \text { a.e } .
$$

If, furthermore, $T(\lambda)$ satisfies (3.7), then we have

$$
\Re\left(U T-I_{r}\right) \subset \mathfrak{R}(T) \text {. }
$$

We shall now construct the second representation of $\mathfrak{M}$. Let $\mathfrak{D}$ be the set of all $C^{r}$-valued measurable functions $g(\lambda) \in C^{r},-\infty<\lambda<\infty$, such that $\left(\Gamma^{\prime}(\lambda) g(\lambda), g(\lambda)\right)_{r}$ is integrable with respect to the Lebesgue measure. Since the matrix $\Gamma^{\prime}(\lambda)$ is Hermitian symmetric and semipositive definite, the sesqui-linear form

$$
\langle g, h\rangle=\int_{-\infty}^{\infty}\left(\Gamma^{\prime} g, h\right)_{r} d \lambda, \quad g, h \in \mathfrak{D}
$$

defined in $\mathfrak{D}$ is semi-positive definite. Let $(B)$ be the set of all residue classes in $\mathfrak{D}$ modulo the subspace consisting of all $g \in \mathfrak{D}$ such that $\langle g, g\rangle=0$. Then, (8) is a pre-Hilbert space in which the inner product of $\mathfrak{g} \in \mathbb{B S}$ and $\mathfrak{h} \in \mathbb{S}$ is defined by (3.13) with $g$ and $h$ being representatives of the residue classes $\mathfrak{g}$ and $\mathfrak{h}$, respectively. The space $\$(S$ will be our second representation space. A representative of $\mathfrak{g} \in \mathbb{B}$ will generally be denoted by $g(\lambda)$.

LeMMA 3.3. Let $T(\lambda)$ be a representative function of $\left(t_{k i}\right)$ defined by (3.6). Then, the mapping $\tau$ which assigns $T(\lambda) g(\lambda)$ to each $\mathfrak{g} \in \mathbb{B}$ is well-defined as a mapping from (B) to $\mathfrak{F}$ and $\tau$ is independent of the choice of representative function $T(\lambda)$. Furthermore, $\tau$ is an isometry with $\Re(\tau)=\mathfrak{F}$, so that (\$S is a (complete) Hilbert space. The inverse of $\tau$ is given by $\left(\tau^{-1} f\right)(\lambda)=U(\lambda) f(\lambda), f \in \mathfrak{F}$, where $U(\lambda)$ is an arbitrary measurable matrix-valued function satisfying (3.11).

Proof. The fact that $\tau$ is a well-defined isometry from $\mathbb{B}$ to $\mathfrak{F}$ is readily seen from (3.8) and the definition of the inner product in (3) (see (3.13)). The relation (3.8) is still true, if one of two $T$ 's on the right-hand side is replaced by another representative function of 
$\left(t_{k i}\right)$. The independence of $\tau$ on the choice of $T(\lambda)$ follows from this at once. Let $f \in \mathfrak{F}$ be arbitrary and $f(\lambda)$ a representative function of $f$. Since (3.8), (3.11), and Lemma 3.1 imply $U^{*} \Gamma^{\prime} U=Q \Sigma^{\prime} Q=\Sigma^{\prime}$, a.e., we see that $g(\lambda)=U(\lambda) f(\lambda)$ belongs to $\mathscr{D}$ and hence $g(\lambda)$ determines a residue class $g \in \mathbb{S}$. On the other hand, it follows from (3.11) and Lemma 3.1 that $\Sigma^{\prime}(T g-f)(\lambda)=0$, a.e. Hence, $\tau \mathfrak{g}=f$ and the proof of the lemma is complete.

Using the mapping $\tau$ given in Lemma 3.3, we now define the mapping $G$ from $\mathfrak{M}$ to $\&$ by putting

$$
G x=\tau^{-1} F x, \quad \text { for each } x \in \mathfrak{M} \text {. }
$$

Then, by Lemma $3.3 G$ is a unitary mapping from $\mathfrak{M}$ onto $(S)$. The inner product of $x$ and $y$ in $\mathfrak{M}$ is given by

$$
(x, y)=\int_{-\infty}^{\infty}\left(\Gamma^{\prime}(\lambda)(G x)(\lambda),(G y)(\lambda)\right)_{r} d \lambda .
$$

For brevity we say that $f(\lambda)=g(\lambda)$ in (S) (or $\mathfrak{F}$ ), if $f(\lambda)$ and $g(\lambda)$ are representative functions of the same element of $\mathbb{B}$ (or $\mathfrak{F}$ ). Then, it is clear that $(G H x)(\lambda)=\lambda(G x)(\lambda)$ in $\mathbb{B}$ for each $x \in \mathfrak{M}$ in the domain of $H$. Thus, we get our second representation of $\mathfrak{M}$.

Lemma 3.3 gives the relation between two representations. Namely, we have

$$
\begin{cases}(G x)(\lambda)=U(\lambda)(F x)(\lambda) & \text { in } \mathfrak{S}, \\ (F x)(\lambda)=T(\lambda)(G x)(\lambda) & \text { in } \mathfrak{F} .\end{cases}
$$

Finally, we note that, if $x \in \mathfrak{M}$ admits the representation of the type (3.5) and if the $r$-tuple formed by $x_{1}(\lambda), \cdots, x_{r}(\lambda)$ is denoted by $x(\lambda)$, then the relation

$$
(G x)(\lambda)=x(\lambda) \text { in } \mathbb{S}
$$

holds true. Indeed, (3.5) and (3.6) imply $(F x)(\lambda)=T(\lambda) x(\lambda)$, so that $(G x)(\lambda)=(U T)(\lambda) x(\lambda)$ by (3.16). However, (3.8), (3.11), and Lemma 3.1 imply that $\Gamma^{\prime}\left(U T-I_{r}\right)=T^{*} \Sigma^{\prime} T U T-\Gamma^{\prime}=0$. Hence, $(U T)(\lambda) x(\lambda)=$ $x(\lambda)$ in $(8)$ and (3.17) is proved.

4. Unitary equivalence of the absolutely continuous spectra. We shall next introduce the main tool for getting the representation of $W_{ \pm}$, that is, the matrix function $W(z)$. As an easy consequence of its basic properties, the "time-independent" proof of the unitary equivalence under consideration will be given at the end of the section.

Throughout this and the following sections, we agree that $H_{0}, V$, and $H_{1}$ are as given in 1 and that all of the notations such as $\beta_{i j}$ introduced in 3 are used with subscript $p$ or superscript $(p), p=1,2$, 
when they refer to $H_{p}$ instead of $H$ in the previous section. In particular, $\mathfrak{M}_{p}$ is the a.c. subspace of $\mathfrak{S}_{\mathfrak{g}}$ with respect to $H_{p}$ and $\mathfrak{F}_{p}$ and $\mathbb{S}_{p}$ are its representation spaces.

For each complex number $z$ belonging to the resolvent set of $H_{p}$, the complex-valued function $w_{i j}^{(p)}(z), 1 \leqq i, j \leqq r$, is defined as

$$
w_{i j}^{(p)}(z)=\delta_{i j}+(-1)^{p}\left(V\left(H_{p}-z\right)^{-1} \varphi_{j}, \varphi_{i}\right) \text {. }
$$

By a simple computation using (1.1) we see that the matrix $W_{p} \in M(r)$ formed by $w_{i j}^{(p)}(z)$ is written as

$$
W_{p}(z)=I_{r}+(-1)^{p} C \int_{-\infty}^{\infty}(\mu-z)^{-1} d B_{p}(\mu),
$$

where $C \in M(r)$ is given by $C=\left\{c_{i} \delta_{i j}\right\}$ with $c_{i}$ given in (1.1). As is well-known (see, e.g. [6, Chapt. 7]), the boundary values on reals of $W_{p}(z)$,

$$
W_{p \pm}(\lambda)=\lim _{\varepsilon \downarrow 0} W_{p}(\lambda \pm i \varepsilon),
$$

exist for almost every $\lambda$. Our arguments will essentially be based upon the following formulas involving $W$ :

$$
\begin{gathered}
W_{p+}(\lambda)-W_{p-}(\lambda)=2 \pi i(-1)^{p} C B_{p}^{\prime}(\lambda), \text { a.e. ; } \\
W_{1}(z)=W_{0}(z)^{-1} ; \quad W_{1 \pm}(\lambda)^{-1}=W_{0 \pm}(\lambda)^{-1}, \quad \text { a.e. } ; \\
W_{p}(z) C=C W_{p}(\bar{z})^{*} ; \quad W_{p \pm}(\lambda) C=C W_{p \mp}(\lambda)^{*}, \text { a.e. } ; \\
C \Gamma_{p}^{\prime}(\lambda)=\left(W_{p \pm} C \Gamma_{q}^{\prime} W_{p \mp}\right)(\lambda),
\end{gathered}
$$

where $q$ is the one of 0 and 1 which is different from $p$.

Proof of (4.3)-(4.6). The $(i, j)$ component of the left-hand side of (4.3) is equal to

$$
\begin{gathered}
(-1)^{p} c_{i} \lim _{\varepsilon \downarrow 0} \int_{-\infty}^{\infty}\left((\mu-(\lambda+i \varepsilon))^{-1}-(\mu-(\lambda-i \varepsilon))^{-1}\right) d \beta_{i j}^{(p)}(\mu) \\
=2 \pi i(-1)^{p} c_{i} \beta_{i j}^{(p) \prime}(\lambda), \quad \text { a.e. }
\end{gathered}
$$

(for the last equality, see, e.g., [6, Chapt. VII]). This proves (4.3). The first of (4.4) follows easily from the fact that

$$
I+V\left(H_{0}-z\right)^{-1}=\left(H_{1}-z\right)\left(H_{0}-z\right)^{-1}=\left(I-V\left(H_{1}-z\right)^{-1}\right)^{-1} .
$$

By multiplying both sides of (4.2) by $C$ from the right, we obtain

$$
\begin{aligned}
W_{p}(z) C & =C\left(1+(-1)^{p} \int_{-\infty}^{\infty}(\mu-z)^{-1} d B_{p}(\mu) C\right) \\
& =C\left(1+(-1)^{p} C \int_{-\infty}^{\infty}(\mu-\bar{z})^{-1} d B_{p}(\mu)\right)^{*},
\end{aligned}
$$


where we used the fact that $C$ and $B_{p}(\mu)$ are Hermitian symmetric. Thus, we get the first of (4.5). The second formulas of (4.4) and (4.5) are obtained from the respective first by taking the limit. By the repeated use of (4.3) and (4.4), we get $2 \pi i(-1)^{p} C B_{p}^{\prime}=W_{q+}^{-1}-W_{q-}^{-1}=$ $W_{q \pm}^{-1}\left(W_{q-}-W_{q+}\right) W_{q \mp}^{-1}=2 \pi i(-1)^{q+1} W_{p \pm} C B_{q}^{\prime} W_{p \mp}$. Hence, (4.6) follows from (3.2).

THEOREM 1. ([2, Theorem 1]) The absolutely continuous parts of $H_{0}$ and $H_{1}$ are unitarily equivalent. ${ }^{3}$

Proof. Since all the matrices in (4.6) except $\Gamma^{\prime}$ are regular, we see that $r\left(\Gamma_{0}^{\prime}(\lambda)\right)=r\left(\Gamma_{1}^{\prime}(\lambda)\right)$, a.e. Consequently, it follows from (3.10) that the multiplicity of $\lambda$ with respect to the a.c. parts of $H_{0}$ and $H_{1}$ coincide with each other a.e. As for the a.c. spectra. the last statement is equivalent to the unitary equivalence ([7, Chapt. VII]).

5. Representation of wave and scattering operators. A representation of $W_{ \pm}$is now obtained, roughly speaking, as the multiplication of $W_{0 \pm}(\lambda)$, which maps $\mathbb{S}_{0}$ onto $\mathbb{B}_{1}$. We shall begin with the following lemma.

LEMma 5.1. The mapping which assigns $h_{ \pm}(\lambda)=W_{p \pm}(\lambda) g(\lambda)$ to each $\mathrm{g} \in \mathbb{S}_{p}$ is well-defined as an isometry from $\mathbb{S}_{p}$ to $\mathbb{B S}_{q}$.

Proof. By virtue of (4.4), (4.5), and (4.6) we get

$$
W_{p \pm}^{*} \Gamma_{q}^{\prime} W_{p_{ \pm}}=C^{-1} W_{p \mp} C \Gamma_{q}^{\prime} W_{p_{ \pm}}=C^{-1} W_{p \mp}\left(W_{q \mp} C \Gamma_{p}^{\prime} W_{q \pm}\right) W_{p_{ \pm}}=\Gamma_{p}^{\prime} \text {, a.e . }
$$

Hence,

$$
\int_{-\infty}^{\infty}\left(\Gamma_{p}^{\prime} h_{ \pm}, h_{ \pm}\right)_{r} d \lambda=\int_{-\infty}^{\infty}\left(\Gamma_{p}^{\prime} g, g\right)_{r} d \lambda
$$

for each $g$. The lemma follows from this at once.

With the aid of this lemma our representation of $W_{ \pm}$and $S$ can now be formulated as follows.

THEOREM 2. Let $G_{p}$ be the isometry from $\mathfrak{M}_{p}$ onto $\mathscr{S}_{p}$ defined by (3.14). Furthermore, let $\boldsymbol{W}_{p \pm}$ be the isometry from $\mathbb{S}_{p}$ to $\mathbb{S}_{q}$ determined in Lemma 5.1. Then, the wave operator defined by (2.1) is expressible as

$$
W_{ \pm}\left(H_{q}, H_{p}\right)=G_{q}^{-1} W_{p \pm} G_{p} P_{p} \text {. }
$$

In particular, $\boldsymbol{W}_{p_{ \pm}}$maps $\mathbb{S}_{p}$ onto $\mathbb{S}_{q}$ and hence has the inverse. The

3 That the theorem remains true without the assumption (1.2) is easily seen from the facts we have remarked just before (1.2). 
scattering operator is expressed as

$$
S=G_{0}^{-1} S G_{0} P_{0}, \quad S=W_{0+}^{-1} W_{0-} .
$$

If we want to use $\mathfrak{F}_{p}$ and $\mathfrak{F}_{q}$ as representation spaces, then we have the following version of Theorem 2, which follows readily from Theorem 2 and (3.14).

Theorem $2^{\prime}$ Let $F_{p}$ be as in 3 and let $\tau_{p}$ be defined as in Lemma 3.3. Then, we have

$$
\begin{gathered}
W_{ \pm}\left(H_{q}, H_{p}\right)=F_{q}^{-1}\left(\tau_{q} \boldsymbol{W}_{p \pm} \tau_{p}^{-1}\right) F_{p} P_{p}, \\
S=F_{0}^{-1}\left(\tau_{0} S \tau_{0}^{-1}\right) F_{0} P_{0} .
\end{gathered}
$$

REMARK 1. Using representative functions, (5.1) and (5.2) can be written as

$$
\left\{\begin{aligned}
\left(G_{q} W_{ \pm} x\right)(\lambda) & =W_{p \pm}(\lambda)\left(G_{p} x\right)(\lambda) & & \text { in } \mathbb{B}_{q}, x \in \mathfrak{M}_{p} \\
\left(G_{0} S x\right)(\lambda) & =K(\lambda)\left(G_{0} x\right)(\lambda) & & \text { in } \mathbb{S}_{0}, x \in \mathfrak{M}_{0}
\end{aligned}\right.
$$

where we put

$$
K(\lambda)=W_{0+}(\lambda)^{-1} W_{0-}(\lambda) \in M(r) .
$$

In the similar formulas corresponding to $(5.1)^{\prime}$ and $(5.2)^{\prime}$, the matrices $W_{p_{ \pm}}$and $K$ in (5.3) should be replaced by $\left(T_{q} W_{p_{ \pm}} U_{p}\right)(\lambda)$ and $\left(T_{0} K U_{0}\right)(\lambda)$, respectively, where $T_{q}$ and $U_{p}$ are as in 3 (in particular, see (3.11)).

REMARK 2. The wave operator $W_{ \pm}\left(H_{q}, H_{p}\right)$ maps $\mathfrak{M}_{p}$ isometrically onto $\mathfrak{M}_{q}$ and furnishes the unitary equivalence considered in 4 (see [3]). Hence, by (5.1) the operator $\hat{W}_{ \pm}\left(H_{q}, H_{p}\right)=G_{q}^{-1} W_{p_{ \pm}} G_{p} P_{p}$ has the same properties. (In particular, therefore, $W_{p \pm}$ maps $\mathbb{S}_{p}$ onto $\mathbb{S}_{q}$. .) These properties themselves, however, can also be proved directly without referring to (5.1). In fact, we easily see $\hat{W}_{ \pm}\left(H_{q}, H_{p}\right)^{*}=\widehat{W}_{ \pm}\left(H_{p}, H_{q}\right)$, which implies $\mathfrak{R}\left(\hat{W}_{ \pm}\left(H_{q}, H_{p}\right)\right)=\mathfrak{M}_{1}$. Furthermore, the definition of $\hat{W}_{ \pm}$gives the unitary equivalence at once.

REMARK 3. In the coming proof of Theorem 2 we shall make use of the already established fact that the limit in (2.1) exists. However, the proof of this fact usually requires a step by step consideration with respect to the rank of $V$ based on the "transitivity" (see, e.g., [5, (1.6)]) of the wave operators. It might therefore be of interest to remark that the existence of that limit, together with the formula (5.1), can be proved directly without referring to the transitivity, so that we have a method of fully time-independent character to treat the whole problem. This can be done for instance, by literally gener- 
alizing the arguments used by Kato [3] for the proof of the case $r=1$.

Proof of Theorem 2. The formula (5.1) implies all the other statements in the theorem (see the first part of Remark 2 and the formula (2.1)). We shall prove (5.1) for $W_{+}=W_{+}\left(H_{1}, H_{0}\right)$. The other cases can be handled similarly.

For simplicity we put

$$
\hat{W}_{+}=G_{1}^{-1} W_{0+} G_{0} P_{0} \text {. }
$$

Since $W_{+}$and $\hat{W}_{+}$are partially isometric operators with the initial set $\mathfrak{M}_{0}$ and the final set contained in $\mathfrak{M}_{1}$, it suffices to prove that $\left(W_{+} x, y\right)=$ $\left(\hat{W}_{+} x, y\right)$ for each $x \in \mathfrak{M}_{0}$ and $y \in \mathfrak{M}_{1}$. Moreover, since the linear hull of $\bigcup_{k=1}^{r} \mathfrak{M}_{p, k}, \mathfrak{M}_{p, k}=\mathfrak{L}\left(P_{p} \varphi_{k} ; H_{p}\right)$ is dense in $\mathfrak{M}_{p}$, it is sufficient to show the above relation for each $x \in \mathfrak{M}_{0, i}$ and $y \in \mathfrak{M}_{1, j}, 1 \leqq i, j \leqq r$. Now, such $x$ and $y$ are expressible as $x=f\left(H_{0}\right) P_{0} \varphi_{i}$ and $y=g\left(H_{1}\right) P_{1} \varphi_{j}$, where $f \in L^{2}\left(\gamma_{i i}^{(0)}\right)$ and $g \in L^{2}\left(\gamma_{j j}^{(1)}\right)$. Therefore, we have

$$
\left(W_{+} x, y\right)=\int_{-\infty}^{\infty} \overline{g(\lambda)} \rho^{\prime}(\lambda) d \lambda,
$$

where we put $\rho(\lambda)=\left(E_{1}(\lambda) W_{+} x, P_{1} \varphi_{j}\right)$. With the aid of certain properties of $W_{ \pm}$(see, e.g., $[5, \S 1]$ ) the function $\rho(\lambda)$ can be computed as follows:

$$
\begin{aligned}
\rho(\lambda) & =\left(W_{+} E_{0}(\lambda) x, P_{1} \varphi_{j}\right)=\left(W_{+} E_{0}(\lambda) x, \varphi_{j}\right) \\
& =\left(E_{0}(\lambda) x, \varphi_{j}\right)+i \lim _{\varepsilon \downarrow 0} \int_{0}^{\infty}\left(e^{i t\left(B_{1}+i \varepsilon\right)} V e^{-i t B_{0}} E_{0}(\lambda) x, \varphi_{j}\right) d t .
\end{aligned}
$$

The integral on the right side is equal to

$$
\begin{aligned}
\sum_{k=1}^{r} c_{k} & \int_{0}^{\infty}\left(e^{-i t H_{0}} E_{0}(\lambda) x, \varphi_{k}\right)\left(e^{i t\left(H_{1}+i \varepsilon\right)} \varphi_{k}, \varphi_{j}\right) d t \\
= & \sum_{k=1}^{r} c_{k} \int_{0}^{\infty} d t \int_{-\infty}^{\lambda} e^{-i t \mu} f(\mu) \gamma_{k i}^{(0) \prime}(\mu) d \mu \int_{-\infty}^{\infty} e^{i t(\nu+i \varepsilon)} d \beta_{j k}^{(1)}(\nu) \\
& =-i \int_{-\infty}^{\lambda} k_{\varepsilon}(\mu) d \mu,
\end{aligned}
$$

where we put

$$
k_{\varepsilon}(\mu)=f(\mu) \sum_{k=1}^{r} \gamma_{k i}^{(0) \prime}(\mu) \int_{-\infty}^{\infty} c_{k}(\mu-\nu-i \varepsilon)^{-1} d \beta_{j k}^{(1)}(\nu) .
$$

As $\varepsilon$ tends to zero from above, $k_{\varepsilon}(\mu)$ tends a.e. to

$$
\left.k(\mu)=f(\mu) \sum \gamma_{k i}^{(0) '}(\mu) \overline{\left(w_{k j}^{(1+)}(\mu)\right.}-\delta_{k j}\right) .
$$

It is easily seen, however, that the set of all $f \in L^{2}\left(\gamma_{i i}^{(0)}\right)$ such that $k_{\varepsilon}$ tend to $k$ in the sense of $L^{1}(-\infty, \infty)$ forms a dense set of $L^{2}\left(\gamma_{i i}^{(0)}\right)$. 
Consequently, the set $\mathscr{R}$ of all those $x \in \mathfrak{M}_{0 i}$, which are expressible as $x=f\left(H_{0}\right) P_{0} \varphi_{i}$ with such $f$ as specified above, forms a dense set of $\mathfrak{M}_{0 i}$.

Let now $x \in \Re$ and substitute the integral in (5.7) by the right side of (5.8). Then, the limit $\varepsilon \downarrow 0$ can be taken under the integral sign with respect to $\mu$, and the limit of the integrand belongs to $L^{1}(-\infty, \infty)$. Hence, by differentiation we get

$$
\rho^{\prime}(\lambda)=f(\lambda) \gamma_{j i}^{(0)}(\lambda)+k(\lambda) \text {. }
$$

By the definition of $k(\lambda)$ the right-hand side of (5.9) is equal to $f(\lambda)$ times the $(j, i)$ element of the matrix $W_{1+}^{*} \Gamma_{0}^{\prime}=\Gamma_{1}^{\prime} W_{0+}$ (the last equality is implied by (4.5) and (4.6)). Thus, we get from (5.6) that

$$
\left(W_{+} x, y\right)=\sum_{k=1}^{r} \int_{-\infty}^{\infty} \gamma_{j k}^{(1) \prime} w_{k i}^{(0+)} f \bar{g} d \lambda .
$$

By (5.5), (3.15), and (3.17) we finally obtain $\left(W_{+} x, y\right)=\left(\hat{W}_{+} x, y\right)$ for each $x \in \Re$ and $y \in \mathfrak{M}_{1 j}$. Since $\Re$ is dense in $\mathfrak{M}_{0 i}$, the proof of the theorem is complete.

6. Diagonalization of scattering operator. Since $S$ is a unitary operator in $\mathfrak{M}_{0}$ which commutes with $H_{0}$, it takes a diagonal form in a certain representation space of the type $\mathfrak{F}$ determined by a special choice of the set $\left\{\psi_{k}\right\}$. Namely, there exists a set $\left\{\psi_{k} ; k=1, \cdots, n\right\} \subset \mathfrak{M}_{0}$, $\mathfrak{M}_{0}=\sum_{k=1}^{n} \oplus \mathfrak{L}\left(\psi_{k} ; H_{0}\right)$, such that, besides the properties (i)-(iv) specified near the beginning of 3 , it has the following additional property: $S$ maps $\mathfrak{Q}\left(\psi_{k} ; H_{0}\right)$ onto itself and is represented there by a multiplicative operator. Precisely speaking, for each $k, 1 \leqq k \leqq n$, there exists $\xi_{k} \in L^{2}\left(\sigma_{k}\right), \sigma_{k}(\lambda)=\left(E_{0}(\lambda) \psi_{k}, \psi_{k}\right)$, such that $\left|\xi_{k}(\lambda)\right|=1$ a.e. with respect to the measure $d \sigma_{k}$ and $x=f\left(H_{0}\right) \psi_{k}$ implies $S x=\xi_{k} f\left(H_{0}\right) \psi_{k}$. The function $\xi_{k}(\lambda)$ may be called the eigenvales of $S$ in the spectral representation space. In what follows we shall show that $\xi_{k}(\lambda)$ are given as eigenvalues of the matrix $K(\lambda)$ defined by (5.4).

THEOREM 3. For almost every $\lambda$ the following statement holds true. Let $m=m(\lambda)$. Then, among $r$ eigenvalues of $K(\lambda)$, there exist $r-m$ eigenvalues 1 , and the remaining $m$ eigenvalues are equal to $\xi_{1}(\lambda), \cdots, \xi_{m}(\lambda)$ in a certain order. (Some of $\xi_{k}(\lambda)$ may be equal to 1.)

Proof. Let us first note the following properties of $W_{p_{ \pm}}$and $K$. Put $\mathfrak{N}_{p}(\lambda)=\mathfrak{N}\left(\Gamma_{p}^{\prime}(\lambda)\right)$. Then, (4.6) implies that

$$
W_{p \pm}(\lambda) \mathfrak{N}_{p}(\lambda)=\mathfrak{N}_{q}(\lambda) \text {, a.e . }
$$

Since $W_{1+}(\lambda)=W_{1-}(\lambda)$ on $\mathfrak{R}_{1}(\lambda)$ by (4.3) and (3.2), it follows from (4.4) and (6.1) with $p=0$ that 


$$
K(\lambda)=I_{r} \text { on } \mathfrak{R}_{0}(\lambda) \text {. }
$$

Let now $\left\{\psi_{k}\right\}$ be one of those "bases" of $\mathfrak{M}_{0}$ with respect to which $S$ has the diagonal form as prescribed above. In reference to $\left\{\psi_{k}\right\}$ thus fixed, let $T_{0}(\lambda)$ and $U_{0}(\lambda)$ be defined as in 3. Furthermore, we can and shall require that $T_{0}(\lambda)$ satisfies (3.7). Then, the following arguments hold true for almost all $\lambda$.

Let $\lambda$ be fixed and let $m=m(\lambda)$. Hereafter, we simply write $T_{0}$ etc. instead of $T_{0}(\lambda)$ etc. By the assumption (3.7), the matrices $T_{0} \in M(n, r)$ and $U_{0} \in M(r, n)$ have the form

$$
\begin{aligned}
& T_{0}=\left[\begin{array}{c}
A \\
0
\end{array}\right], \quad A \in M(m, r) \\
& U_{0}=(B, C), \quad B \in M(r, m), \quad C \in(r, n-m) .
\end{aligned}
$$

Here, $n-m$ column vectors of $C$ belong to $\mathfrak{N}_{0}$ and $A B=I_{m}$ because of (3.11). (Note that the assumption (3.7) implies $\mathfrak{N}\left(T_{0}\right)=\mathfrak{R}_{0}$ because of (3.8).) It therefore follows from (6.2) that the matrix $J=T_{0} K U_{0} \in M(n)$ has the form

$$
J=T_{0} K U_{0}=\left[\begin{array}{cc}
A K B & 0 \\
0 & 0
\end{array}\right], \quad A K B \in M(m) .
$$

We note that $J$ is the matrix introduced in Remark 1 after Theorem $2^{\prime}$. Hence, in view of that remark and the special choice of $\left\{\psi_{k}\right\}$ made above, we thus obtain

$$
\left\{\begin{array}{l}
A K B \text { is the diagonal matrix with the } \\
\text { diagonal elements } \xi_{1} \cdots, \xi_{m} \text {. }
\end{array}\right.
$$

Since the only restriction imposed on $U_{0}$ is (3.11), we have much freedom in choosing it. In particular, since (3.7) and (3.9) implies $\Re\left(T_{0}\right)=C_{n}^{m}$, we can make $U_{0} C_{n}^{m}$ equal to the orthogonal complement $\mathfrak{R}_{0}^{\prime}$ of $\mathfrak{R}_{0}$. In such a choice of $U_{0}$, all the $m$ column vectors of $B$ span $\mathfrak{N}_{0}^{\prime}$. Let now $D \in M(r, r-m)$ be a matrix such that $r-m$ column vectors of $D$ form a complete orthonormal set of $\mathfrak{R}_{0}$. Then, the matrix $Z=$ $(B, D) \in M(r)$ is a regular matrix with the inverse $Z^{-1}=\left[\begin{array}{l}A \\ D^{*}\end{array}\right]$ (note that $A B=I_{m}$ ). Using (6.2) and the special feature of $B$ and $D$, we finally obtain

$$
Z^{-1} K Z=\left[\begin{array}{cc}
A K B & 0 \\
D^{*} K B & I_{r-m}
\end{array}\right],
$$

which proves the theorem in virtue of (6.3).

REMARK. If $\xi_{k}(\lambda) \neq 1$, a.e., with respect to $d \sigma_{k}$, we can easily 
show that a representative function of $F_{0} \psi_{k} \in \widetilde{F}_{0}$ is the eigenvector of $K(\lambda)$ with the eigenvalue $\xi_{k}(\lambda)$. However, we do not know if $\xi_{k}(\lambda) \neq 1$ a.e. with respect to $d \sigma_{k}$ or not. For this reason another proof of Theorem 3 along this line, which is actually possible, may not be trivially simple.

In this connection we observe that $S x=x, x \in \mathfrak{M}_{0}$, means $C \Gamma_{1}^{\prime} W_{0-} F_{0} x \in \mathfrak{R}\left(\Gamma_{0}^{\prime}\right)$ (see (4.3)). If $r=1$, it is impossible (under the assumption of (1.2)). A stronger requirement that $S=I$ on $\mathfrak{M}_{0}$ makes $\Re\left(C \Gamma_{1}^{\prime} W_{0-}\right) \subset \Re\left(\Gamma_{0}^{\prime}\right)$ necessary and the latter is possible only when $r \geqq 2 n$. However, we cannot say anything more about this uniqueness problem of $S$ at present.

Added in proof. Using the ideas developed by L. de Branges (L. de Branges, Perturbation of self-adjoint transformations, Amer. J. Math., 84 (1962), 543-560), some results in the present paper will be shown to have their analogues for a wider class of perturbations. This will be discussed elsewhere.

\section{REFERENCES}

1. M. S. Birman and M. G. Krein, On the theory of wave operators and scattering operators, Dokl. Akad. Nauk, S.S.S.R., 144 (1962), 475-478.

2. T. Ikebe, Eigenfunction expansions associated with the Schroedinger operators and their applications to scattering theory, Arch. Rat. Mech. Anal., 5 (1960), 1-34.

3. T. Kato, On finite-dimensional perturbations of self-adjoint operators, J. Math. Soc. Japan, 9 (1957), 239-249.

4. S. T. Kuroda, On a generalization of Weinstein-Aronszajn formula and the infinite determinant, Sci. Papers of College of General Education, Univ. of Tokyo, 11 (1961), 1-12. 5. - Perturbation of continuous spectra by unbounded operators, I, J. Math. Soc. Japan, 11 (1959), 247-262.

6. R. H. Nevanlinna, Eindeutige analytische Funktionen, Berlin, (1936).

7. M. H. Stone, Linear transformations in Hilbert space and their applications to analysis, Amer. Math. Soc. Coll. Publ., 15, New York (1932).

UNIVERSITY OF CALIFORNIA, BERKELEY

AND

UNIVERSITY OF TOKYO 


\section{PACIFIC JOURNAL OF MATHEMATICS}

\section{EDITORS}

RalPh S. Phillips

Stanford University

Stanford, California

M. G. Arsove

University of Washington

Seattle 5 , Washington
J. Dugundu

University of Southern California

Los Angeles 7, California

Lowell J. Paige

University of California

Los Angeles 24, California

\section{ASSOCIATE EDITORS}
E. F. BECKENBACH
D. DERRY
H. L. ROYDEN
E. G. STRAUS
T. M. CHERRY
M. OHTSUKA
E. SPANIER
F. WOLF

\section{SUPPORTING INSTITUTIONS}

\author{
UNIVERSITY OF BRITISH COLUMBIA \\ CALIFORNIA INSTITUTE OF TECHNOLOGY \\ UNIVERSITY OF CALIFORNIA \\ MONTANA STATE UNIVERSITY \\ UNIVERSITY OF NEVADA \\ NEW MEXICO STATE UNIVERSITY \\ OREGON STATE UNIVERSITY \\ UNIVERSITY OF OREGON \\ OSAKA UNIVERSITY \\ UNIVERSITY OF SOUTHERN CALIFORNIA
}

\author{
STANFORD UNIVERSITY \\ UNIVERSITY OF TOKYO \\ UNIVERSITY OF UTAH \\ WASHINGTON STATE UNIVERSITY \\ UNIVERSITY OF WASHINGTON \\ AMERICAN MATHEMATICAL SOCIETY \\ CALIFORNIA RESEARCH CORPORATION \\ SPACE TECHNOLOGY LABORATORIES \\ NAVAL ORDNANCE TEST STATION
}

Mathematical papers intended for publication in the Pacific Journal of Mathematrcs should be typewritten (double spaced), and the author should keep a complete copy. Manuscripts may be sent to any one of the four editors. All other communications to the editors should be addressed to the managing editor, L. J. Paige at the University of California, Los Angeles 24, California.

50 reprints per author of each article are furnished free of charge; additional copses may be obtained at cost in multiples of 50 .

The Pacific Journal of Mathematics is published quarterly, in March, June, September, and December. Effective with Volume 13 the price per volume (4 numbers) is $\$ 18.00$; single issues, $\$ 5.00$. Special price for current issues to individual faculty members of supporting institutions and to individual members of the American Mathematical Society: $\$ 8.00$ per volume; single issues $\$ 2.50$. Back numbers are available.

Subscriptions, orders for back numbers, and changes of address should be sent to Pacific Journal of Mathematics, 103 Highland Boulevard, Berkeley 8, California.

Printed at Kokusai Bunken Insatsusha (International Academic Printing Co., Ltd.), No. 6 , 2-chome, Fujimi-cho, Chiyoda-ku, Tokyo, Japan.

\section{PUBLISHED BY PACIFIC JOURNAL OF MATHEMATICS, A NON-PROFIT CORPORATION}

The Supporting Institutions listed above contribute to the cost of publication of this Journal, but they are not owners or publishers and have no responsibility for its content or policies. 


\section{Pacific Journal of Mathematics}

\section{Vol. 13, No. $4 \quad$ June, 1963}

Dallas O. Banks, Bounds for eigenvalues and generalized convexity ........... 1031

Jerrold William Bebernes, A subfunction approach to a boundary value problem for

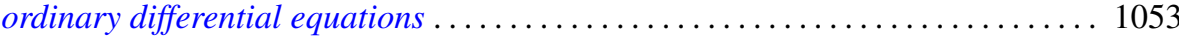

Woodrow Wilson Bledsoe and A. P. Morse, A topological measure construction . . . 1067

George Clements, Entropies of several sets of real valued functions . . . . . . . . . 1085

Sandra Barkdull Cleveland, Homomorphisms of non-commutative *-algebras . . . . . 1097

William John Andrew Culmer and William Ashton Harris, Convergent solutions of

ordinary linear homogeneous difference equations . . . . . . . . . . . . . . . 1111

Ralph DeMarr, Common fixed points for commuting contraction mappings . . . . . . 1139

James Robert Dorroh, Integral equations in normed abelian groups . . . . . . . . 1143

Adriano Mario Garsia, Entropy and singularity of infinite convolutions . . . . . . . 1159

J. J. Gergen, Francis G. Dressel and Wilbur Hallan Purcell, Jr., Convergence of extended Bernstein polynomials in the complex plane ................. 1171

Irving Leonard Glicksberg, A remark on analyticity of function algebras . . . . . . 1181

Charles John August Halberg, Jr., Semigroups of matrices defining linked operators

with different spectra ................................. 1187

Philip Hartman and Nelson Onuchic, On the asymptotic integration of ordinary

differential equations . . . . . . . . . . . . . . . . . . . . . . . . . . . . 1193

Isidore Heller, On a class of equivalent systems of linear inequalities . . . . . . . . . 1209

Joseph Hersch, The method of interior parallels applied to polygonal or multiply

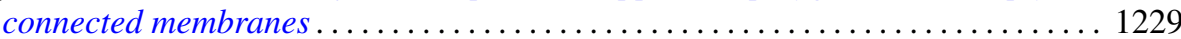

Hans F. Weinberger, An effectless cutting of a vibrating membrane . . . . . . . . . . 1239

Melvin F. Janowitz, Quantifiers and orthomodular lattices ....

Samuel Karlin and Albert Boris J. Novikoff, Generalized convex inequalities . .

Tilla Weinstein, Another conformal structure on immersed surfaces of negative

curvature.

Gregers Louis Krabbe, Spectral permanence of scalar operators

Shige Toshi Kuroda, Finite-dimensional perturbation and a representaion of

scattering operator.

Marvin David Marcus and Afton Herbert Cayford, Equality in certain

inequalities

Joseph Martin, A note on uncountably many disks .

Eugene Kay McLachlan, Extremal elements of the convex cone of semi-norms . . . . 1335

John W. Moon, An extension of Landau's theorem on tournaments . .

Louis Joel Mordell, On the integer solutions of $y(y+1)=x(x$

Kenneth Roy Mount, Some remarks on Fitting's invariants .....

Miroslav Novotný, Über Abbildungen von Mengen ............

Robert Dean Ryan, Conjugate functions in Orlicz spaces.

John Vincent Ryff, On the representation of doubly stochastic operators . . . . . . . . 1379

Donald Ray Sherbert, Banach algebras of Lipschitz functions .

James McLean Sloss, Reflection of biharmonic functions across analytic boundary

conditions with examples.

L. Bruce Treybig, Concerning homogeneity in totally ordered, connected topological space....

John Wermer, The space of real parts of a function algebra...

James Juei-Chin Yeh, Orthogonal developments of functionals and related theorems

in the Wiener space of functions of two variables......... 